\title{
ESTADO DEL ARTE Y REFLEXIONES SOBRE LA DOCUMENTACIÓN ETNOGRÁFICA Y LINGÜÍSTICA DE LAS SOCIEDADES MUINANE Y KOREGUAJE DEL NOROESTE AMAZÓNICO
}

\author{
Consuelo de Vengoechea \\ Universidad Nacional de Colombia \\ mcder@unal.edu.co \\ Camila Sofía Venegas \\ Universidad Nacional de Colombia \\ csvenegaso@unal.edu.co \\ Christian Andrés Cárdenas \\ Universidad Nacional de Colombia \\ chacardenasca@unal.edu.co
}

Recibido: 15/02/2019 - Aprobado: 09/05/2019

DOI: doi.org/10.17533/udea.lyl.n76a01

\begin{abstract}
Resumen: El presente artículo busca realizar un balance sobre la documentación disponible relacionada con la historia, la lengua y la vida social de los pueblos muinane y koreguaje. La metodología empleada se basó en dos ejes principales. El primero fue la pesquisa bibliográfica, la cual permitió la consulta de materiales diversos y representativos sobre la historia, la etnografía, la lengua y situación actual de los grupos sociales muinane y koreguaje. El segundo eje tuvo la intención de reflexionar críticamente sobre ese tipo de materiales y su utilidad dentro de los procesos de fortalecimiento lingüístico y cultural de ambas comunidades.
\end{abstract}

Palabras clave: muinane; koreguaje; documentación; antropología lingüística; revitalización.

\section{STATE OF THE ART AND REFLECTIONS ON THE ETHNOGRAPHIC AND LINGUISTIC DOCUMENTATION OF THE MUINANE AND KOREGUAJE SOCIETIES OF THE AMAZONIAN NORTHWEST}




\begin{abstract}
This article aims at evaluating the available documentation related to the history, language and social life of the Muinane and the Koreguaje peoples. The methodology used was based on two main axes. The first one was the bibliographical research, which allowed the inquiry of diverse and representative materials on the history, ethnography, language, and current situation of the Muinane and Koreguaje communities. The second axis intended to reflect critically about such materials and their usefulness within the linguistic and cultural strengthening processes of both communities.
\end{abstract}

Key words: Muinane; Koreguaje; documentation; linguistic anthropology; revitalization.

\title{
1. Introducción
}

$\dot{i}$ Qué tipo de documentación se tiene a disposición para el conocimiento de las sociedades indígenas muinane del medio Amazonas y koreguaje del Piedemonte Amazónico? Al hacerse la pregunta sobre la documentación a disposición para las sociedades muinane y koreguaje, es de interés abordar el estado del arte sobre dos grupos indígenas que han resistido de manera diferencial a procesos de globalización y conflictos armados en el territorio colombiano, cuyas secuelas transformaron su vida social. No se cuenta con un balance sobre la documentación a disposición en el ámbito histórico, lingüístico y social de los pueblos muinane y koreguaje, lo que sugirió llevar a cabo una revisión de los estudios existentes sobre su historia y aspectos de su vida social, especialmente sus códigos de comunicación. Ante esta realidad, surge la necesidad de crear nueva documentación etnográfica y lingüística que pueda ser útil tanto en la profundización de los conocimientos que existen sobre estos pueblos como en las reivindicaciones sociales y de fortalecimiento cultural, social y lingüístico que reclaman.

El nuevo conocimiento abrirá la posibilidad de llevar a cabo comparaciones entre las sociedades tucano occidental, de una parte, y las bora, de otra. Los registros que se identificaron son de cuatro tipos: bibliográficos, visuales, audiovisuales y de audio. Adicionalmente, se propone una discusión sobre los problemas de interés para la sociedad muinane en particular, y para las sociedades Amazónicas en general, que consisten en entender sus reivindicaciones sociales y políticas a partir del conocimiento que se ha venido construyendo históricamente y del uso y práctica de sus códigos comunicativos: sus lenguas nativas. Estas disquisiciones remiten a 
problemas de antropología lingüística y social que son también trabajados desde las teorías sobre la vitalidad y muerte de lenguas y a partir de procesos de fortalecimiento y la revitalización lingüística. En este punto, se abordan las contradicciones y retos que trae consigo la revitalización lingüística y cultural en un país como Colombia.

\section{Estudios históricos, etnográficos y lingüísticos a disposición sobre las sociedades muinane del medio Amazonas y koreguaje del Piedemonte Amazónico}

\subsection{Muinane o la Gente del Asiento Verde de Centro}

Los muinane hacen parte de un conglomerado de grupos indígenas que se identifican como Gente de Centro en el habla propia: фê:nemuna? $a^{1}$. Aunque hablan lenguas distintas, mantienen relaciones socioculturales constantes y conviven dentro de la región Amazónica, concretamente en los resguardos Aménani, Monochoa y Predio Putumayo. Su territorio tradicional está bordeado por los ríos Caquetá y Putumayo en una extensísima zona de aproximadamente seis millones de hectáreas. En la actualidad se encuentran varios asentamientos muinane en los bordes del río Caquetá, aunque algunos de ellos han migrado a las cabeceras municipales y corregimientos de la región Amazónica, del Departamento del Caquetá y de otras regiones de Colombia, incluyendo hacia Bogotá. La población censada por Vengoechea (2012, p. 26) en el territorio amazónico, sin incluir habitantes de las cabeceras municipales, fue de 259 personas, hecho que plantea el estado de vulnerabilidad de dicho grupo indígena. Los datos oficiales del DANE no se tuvieron a disposición hasta 2017, puesto que los muinane no aparecieron en el Censo DANE $2005^{2}$, lo que constituye un hecho insólito en lo que corresponde a las obligaciones del Estado con sus ciudadanos. De acuerdo con $\mathrm{ACNUR}^{3}$, la población censada en 2005 por el DANE registró la existencia de 87 pueblos indígenas, mientras que la Organización Nacional Indígena de Colombia ONIC indica que son 102, de los cuales 18 están en peligro de

1. Escritura en fonología muinane propuesta a partir del Alfabeto Fonético Internacional por Vengoechea (2012).

2. https://www.dane.gov.co/files/censos/libroCenso2005nacional.pdf

3. https://www.acnur.org/t3/pueblos-indigenas/pueblos-indigenas-en-colombia/

LINGÜÍSTICA Y LITERATURA

18

ISSN 0120-5587

E-ISSN 2422-3174

N. ${ }^{\circ} 76,2019,17-45$ 
desaparecer ${ }^{4}$. El grupo muinane es un ejemplo de este fenómeno de invisibilización, como lo reconoció el Ministerio del Interior ${ }^{5}$, el cual ya poseía un censo muinane que fue elaborado por las mismas comunidades, y que a su vez fue entregado al Estado. Además, varios autores, incluidos Arango y Sánchez (2004), en el Censo Nacional del DANE 2005 y en el informe publicado por ACNUR, registran a los muinane dentro de los uitoto, con quienes se diferencian claramente como se ha podido comprobar en distintas publicaciones científicas (Echeverri y Cruz, 2016).

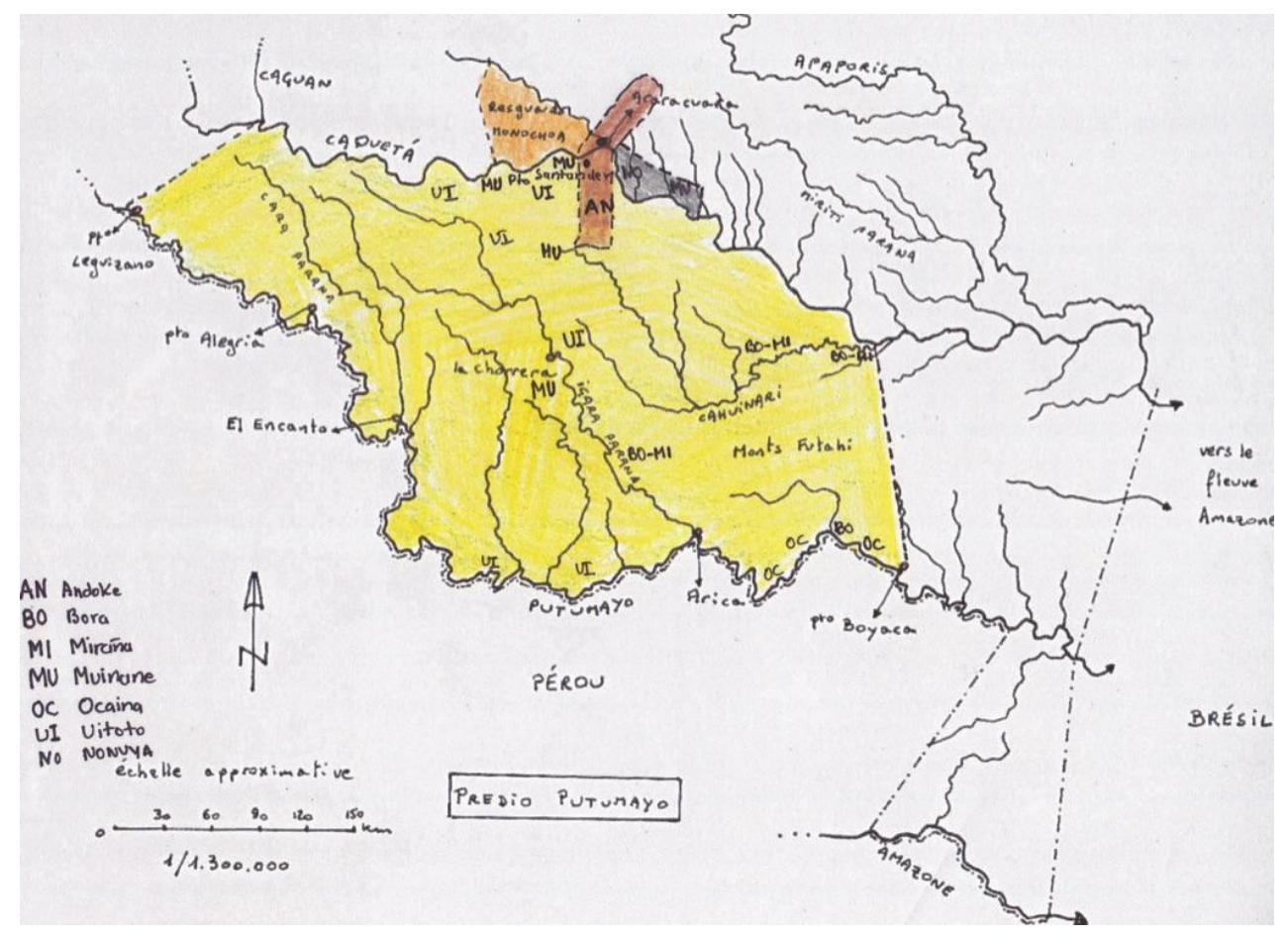

Figura 1. Actuales asentamientos muinane. Vengoechea (2012). Mapa elaborado por el geógrafo Alaon Bunge.

La documentación académica sobre los muinane y su lengua es escasa. Las primeras referencias en la literatura académica sobre los muinane y demás grupos vecinos de la región del Gran Caquetá, específicamente de Araracuara, aparecen bajo el enfoque del estudio de sus lenguas a mediados del siglo XIX, cuando entre 1817 y 1820 Friedrich Von Martius realizó su

4. https://www.todacolombia.com/etnias-de-colombia/grupos-indigenas/distribucion.html 5. http://siic.mininterior.gov.co/sites/default/files/upload/SIIC/PueblosIndigenas/pueblo_muinane.pdf 
expedición por el río Japurá (en Colombia río Caquetá) hasta el raudal de Araracuara, este último límite de dominio portugués en la Colonia. En su libro Beiträge zur Ethnographie und Sprachenkunde Amerika's zumal Brasiliens, de 1867, hace alusión en repetidas ocasiones a los miranhas, cercanos a los muinanes. Posteriormente, en 1904, el etnógrafo Koch Grünberg hizo presencia igualmente en el territorio y realizó también una descripción de los mirañas, en la cual reconoció no haber podido clasificar las lenguas muinane, bora ni andoke (Koch-Grünberg Vol. 2, 1995, p. 286-287). En 1920, Constantin Tastevin logró conformar un vocabulario muinane, el cual clasificó como un dialecto de los miranha, también denominados bora, sin ofrecer los criterios de clasificación (Landaburu, 233-271, 1996).

En cuanto a la clasificación de la lengua, la documentación muestra que han habido varios esfuerzos, como los de Loukotka (1968), quien identificó tres grupos: de lenguas de tribus paleo americanas, de tribus de selvas tropicales y de tribus de los Andes. Loukota ubica en la segunda división las ramas tukano, uitoto, bora, makú, katukina y las lenguas aisladas andoke y yurí. La familia bora es clasificada de la siguiente manera en Loukotka (1968, 190):

- La lengua bora, meaamuyna o miraña, hablada sobre las riberas del río Cahuinarí, del Jacaré y en los montes Futahy, en territorio colombiano y sus tres dialectos: bora o boro, imihita y fa:ai.

-El nonuya hablado en las quebradas del Cahuinarí.

-El muinane, hablado a lo largo del río Arucu.

Posteriormente, Tovar y Larrucea de Tovar (1984, pp. 148-149) distinguieron las dos lenguas y observan que no se debe confundir el idioma muinane-uitoto con el muinane-bora. Una investigación realizada por Ortiz (1986, 128), sobre la documentación existente hasta 1965, permite que Patiño Roselli identifique una etnia de cerca de mil doscientos miembros en el Igará Paraná, bajo Putumayo que habla la lengua bora. Esta lengua tendría varias formas dialectales: bora, miraña, imihita, fa-ai, muinane-bora y nonuya-bora. Aschmann (1993), con base en consideraciones léxicas y gramaticale, considera que los dialectos como el muinane, miraña y bora, pertenecerían a la familia uitoto. Dixon y Aikhenvald (1999, p. 312) aceptaron la propuesta de Aschmann, pero no se limitan en los anteriores. Por su parte, Jon Landaburu (2000) propuso estudiar cuidadosamente las descripciones de las lenguas bora y uitoto. Recientemente, Seifart y Echeverri (2015) proporcionaron una base para la reevaluación de la relación genealógica del 
Bora-Muinane con las lenguas Witoto y Ocaina, propuestas anteriormente por Aschmann (1993), abriendo así la posibilidad de buscar relaciones genealógicas con otras lenguas. La clasificación propuesta por Landaburu (2000, p. 25, 40-41) propuso como lenguas vivas de familia bora tres lenguas: la muinane-bora, de la sabana del Cahuinarí; el bora propiamente dicho, con su muy cercana variante, el miraña; y la lengua miraña-oira-assú-tapuyo.

Con relación a la descripción de la lengua muinane se conocen algunos trabajos que pretenden dar cuenta de su gramática y su fonología, entre ellos los realizados por Vengoechea (1995; 1996a; 1996b; 1999; 2001; 2004-2005; 2003; 2012; 2014). Además, los estudios de Walton, J. (1977), Walton, J., Hensarling, G. y Maxwell M. B. (2000), Walton, J., Pakky, C. y Staly, A. (1978), Walton, J. \& Walton, J. (1967), Walton J. W., Walton J. \& Pakky, C. (1997). Minor, E. E. y de Minor, H. compiladores (1971).

El Instituto Lingüístico de Verano (ILV) promovió actividades para los muinane en 1964 con la ayuda de los lingüistas James y Janice Walton. Durante el periodo de tiempo en el que tuvieron presencia en la región —en colaboración con Edgar Buenaventura y Clementina Pakky - desarrollaron una serie de cartillas orientadas a la alfabetización de la lengua, con las cuales se buscaba apoyar la escuela. Entre algunos títulos de esta serie se encuentran: imuhu ${ }^{6}$ (sal en muinane), mikki iimaamo ${ }^{7}$ (cómo dicen en muinane) y niija ${ }^{8}$ (explicaciones sobre la lluvia). Estas publicaciones se caracterizan por un contenido que se limita a ser el resultado de un ejercicio de transcripción y traducción de un texto, sin que logren representar de forma exitosa y coherente un ejercicio de investigación sobre el conocimiento propio del muinane, que concuerde tanto con las necesidades de la comunidad, como con las especificidades humanas y ambientales de la región. El ILV también realizó la traducción en muinane de un resumen del Antiguo Testamento, del Nuevo Testamento y de un himnario. En relación con el estudio de la lengua, el ILV elaboró una gramática y un diccionario bilingüe muinane-español españolmuinane (Walton, 1997).

En relación con los trabajos de etnografía de los muinane e historia de la región se encuentran los de Koch Grünberg, T. (1909), Londoño Sulkin, C. D. (2004), Llanos, H. y Pineda, R. (1982),

6. Escritura propuesta por Buenaventura y Pakky.

7. Ibídem.

8. Ibídem. 
Pineda Camacho, R. (2000), Gómez, A. (2010), Echeverri, J. A. (1992; 1997; 2016), Useche Losada, M. (1994; 1998), Tobón, M. (2008 y 2018) y Nejedeka, A. (2011).

Roberto Pineda Camacho (2000) intentó reescribir una versión de la historia social de la Casa Arana, en la que muestra cómo la región Amazónica ha sido a través del tiempo concebida por poblaciones no indígenas para la extracción de diversas materias primas, de modo que cada bonanza ha replicado métodos similares de explotación contra las poblaciones indígenas. Así, la Casa Arana es otro de los hitos que han marcado la región al igual que las organizaciones propias de explotación y expansión, con sus modalidades extractivas del caucho y su posterior impacto en la vida social, económica y nativa de las comunidades. Dentro de dicho relato, las narraciones indígenas cobran vital importancia, entretejidas con cotejos de fuentes $\mathrm{y}$, en general, con métodos usados por la historia, como en el caso de la cauchería, practicada por la Casa Arana a finales del siglo XIX.

La anterior reconstrucción de la historia social es un hecho que marcó profundamente las comunidades y fue el punto de partida para que Echeverri (1997) realizara en su etnografía un acercamiento a la manera en que los grupos indígenas pertenecientes a la Gente de Centro han lidiado con los eventos de su historia. Dentro de estos eventos se encuentran procesos como el ingreso de la tecnología de las herramientas metálicas, el genocidio cauchero, la cristianización o el conflicto colombo-peruano. A partir de estos procesos se configura, según Echeverri, una «filosofía del hacha» y de la «palabra de tabaco y coca», fundamentales como expresión de la gente de centro, que propician la reconfiguración de los grupos a partir de la construcción de nuevas formas de identidad colectiva. Igualmente, Echeverri edita en 2017 un texto de nombre Fééne fívo játyime iyáachimihai jíinije ${ }^{9}$ Territorio primordial de vida de la descendencia del Centro: Memorias del territorio del Pueblo Fééneminaa ${ }^{10}$ Gente de Centro, en el cual los Ancianos del pueblo Fééneminaa — nombre con el cual los muinane se autoreconocen en el texto-, expresan sus preocupaciones respecto a la profundización en el conocimiento e interés hacia la cultura y el territorio propio. En el libro, a través de relatos orales, se promulgan la ley del manejo del territorio, al igual que la historia de sus clanes, linajes y territorios.

9. Ortografía aceptada por los muinane y utilizada por Echeverri (2017).

10. Ibídem.

LINGÜÍSTICA Y LITERATURA

ISSN 0120-5587

E-ISSN 2422-3174

N. ${ }^{\circ} 76,2019,17-45$ 
Un trabajo dedicado a los conocimientos propios es realizado por Aniceto Nejedeka $L a$ ciencia de vida escrita en las aves (2011), el cual recoge algunos principios de la creación del mundo para los muinane, principalmente los referentes al acervo sobre las aves. El relato brinda claves para entender la «ciencia de vida» ordenada y escrita para que la humanidad viva bien con su medio, pues como bien está descrita en la obra:

Allí está guardado el conocimiento de las conjuras, allí se aprende a manejar un territorio, allí está el origen de los clanes y linajes, allí se explica la palabra metafórica que se utiliza en los rituales de baile, allí se da cuenta de ciertas profecías (Nejedeka, 2011, p. 3).

Textos como el anterior incursionan en nuevas maneras de transmisión del conocimiento plasmando la enseñanza oral en la escritura, pero operando bajo el mismo principio: ordenar el gran canasto del conocimiento y la creación para entregarlo a nuevas generaciones. Los trabajos abren un camino hacia formas participativas de investigación y de prácticas, en el sentido que surgen de preocupaciones particulares de miembros de la comunidad, quienes en un trabajo colaborativo emprenden un proyecto encaminado a la revaloración del conocimiento propio: la palabra, la lengua y los saberes.

Respecto a la documentación etnográfica del pueblo muinane, uno de los trabajos más sobresalientes es el de Londoño Sulkin (2004), quien en su texto Muinane. Un proyecto moral a perpetuidad, realizó una abstracción e interpretación sintética de las historias contadas por los actores sociales, las cuales, afirma el autor, son las que posibilitan experimentar su mundo. El texto de Londoño contribuye a ampliar el entendimiento de cómo se constituye lo social a través de la subjetivación de los muinane sobre sus propias experiencias. Este se inserta en el debate académico de la antropología amazónica donde nociones como la convivencia, la socialidad, la agencialidad, lo secular y lo doméstico parecen adquirir importancia particularmente en lo que corresponde al estudio de asuntos tales como la reproducción cosmológica, la vida cotidiana y la constitución física y moral de las personas.

Partiendo desde otro enfoque de la etnografía amazónica, el cual tiende a enfatizar en aspectos altamente institucionalizados como el orden mítico, las estructuras sociales y el chamanismo se circunscriben las investigaciones de Fernando Urbina, quien trabajó intensamente con el Abuelo José García, de la nación muinane, y con quien recopiló diversos relatos mitológicos que versan sobre el origen de la coca, del tabaco, de los petroglifos, del 
maguaré, entre otros, los cuales poseen profundos contenidos morales que sugieren sean interiorizados para llegar a tener un buen vivir. Es igualmente destacable el acervo visual que recopiló a lo largo de sus viajes, documentando prácticas relacionadas con los mitos que recogió, relacionando así, a través de sus distintas exposiciones de fotografía o de sus poemas escritos, los relatos recogidos por él a lo largo de su vida.

En cuanto a la documentación audiovisual existente sobre el muinane, una parte proviene de la investigación de Vengoechea (2012) en la cual utilizó materiales de audio de 33,1 horas de grabaciones en el sistema analógico y de 4,08 horas en el sistema digital. En estos registros se encuentran desde listas de palabras y tipologías de saludos hasta cuentos, relatos y canciones. Además, existen cerca de 500 fotografías analógicas, de las cuales 50 han sido digitalizadas. En 2018, Vengoechea, Venegas y Cárdenas adelantan el proyecto de investigación Construcción de memoria y prácticas propias de uso y fortalecimiento de la lengua muinane en la Amazonia colombiana. Fase I, el cual contempla la elaboración de documentación etnográfica y lingüística. El inventario alcanzado en la primera parte de este proceso muestra la documentación audiovisual y de audio de hablantes, hombres y mujeres de diferentes edades y roles sociales, realizando relatos y cantando canciones para los niños y jóvenes.

Respecto a procesos de revitalización lingüística, no se encontraron documentos recientes aparte de una serie de cartillas o textos, como la ya citada por Echeverri (2017) que dan cuenta de la necesidad que la comunidad tiene de estos. Existe, además, el intento de revisión del alfabeto de 1994 de parte del Chukíki o el Consejo de Ancianos de los tres grupos muinanes. Lo anterior indica la carencia de este tipo de trabajo, por lo que se requiere adelantar procesos de fortalecimiento cultural y lingüístico.

\subsection{Koreguaje}

El pueblo koreguaje habita en las riberas y afluentes de los ríos Orteguaza y Caquetá, en donde se encuentra concentrada la mayoría de la población. La lengua koreguaje pertenece a la familia lingüística tukano occidental, y es hablada por personas que se denominan koreguajes, tamas o carijonas (Gralow, 2001, p. 5). Se estima que de los 1767 habitantes pertenecientes al pueblo koreguaje, 1384 son hablantes de la lengua. Esto quiere decir que alrededor de un $80 \%$ 
de las personas se comunican en el idioma nativo. No obstante, esta ha sido catalogada como una lengua en estado de vulnerabilidad según el Ministerio de Cultura, ${ }^{11}$ debido a que pese a ser hablada mayoritariamente, el pueblo en sí mismo presenta un bajo número de personas debido a que ha sufrido las dinámicas del conflicto armado. Además, las competencias de lectoescritura en lengua koreguaje aún no están altamente extendidas en toda la población. Igualmente, el Ministerio de Cultura afirma que «la gran mayoría de los habitantes habla su lengua nativa, desde los abuelos hasta los niños pequeños. Incluso cuando hay visitas de autoridades del Estado o funcionarios indígenas» Por tal motivo, se dice que la actitud general del pueblo frente a su lengua es buena, puesto que existe la voluntad de transmitirla a los niños del mismo modo que lo hacen con el conjunto de sus conocimientos a través de las prácticas cotidianas, como el cuidado de la chagra, los consejos, el relato de historias, los bailes, los cantos, la elaboración de artesanías, la pesca, la caza, entre otras.



Figura 2. Comunidades Koreguaje del Piedemonte amazónico, elaborado por Christian Cárdenas Carrillo.

11. http://www.mincultura.gov.co/areas/poblaciones/APP-de-lenguasnativas/Documents/Estudios\%20Coreguaje.pdf 
Para entender la anterior situación, hay que reflexionar a priori sobre los múltiples procesos políticos, sociales y culturales que han alterado entre tantos aspectos las prácticas de uso y transmisión de las lenguas de numerosos grupos indígenas de la Amazonia y de las cuales el pueblo koreguaje no es una excepción. Su caso, no obstante, debe vislumbrarse a través de sus particularidades, pues las mismas serán determinantes para comprender la coexistencia de dos realidades: por una parte, el territorio de los koreguaje ha sido epicentro del conflicto entre los agentes estatales, paraestatales, las guerrillas y el narcotráfico, al estar situado en las riberas de los ríos Caquetá, Caguán y Orteguaza; por otra parte, la gran mayoría de los koreguaje habla su lengua y conservan una arraigada tradición chamánica.

Tras el contacto, la historia de la región de los koreguaje ha estado marcada por una serie de etapas caracterizadas por los procesos de evangelización, colonización y las economías extractivas a manos de diversos actores. Podría distinguirse a grandes rasgos una primera etapa de colonización misionera, la cual inicia en 1542 con la llegada de misioneros europeos a la región del alto Caquetá y sus primeras fundaciones; la etapa de explotación de caucho y quina, en la primera mitad del siglo Xx; la etapa de colonización a partir de la década de los 40, cuando se muestra a la región como lugar de acogida y refugio para los colonos y se amplían las fronteras agraria y ganadera; y finalmente, el conflicto reciente, caracterizado por la incursión del narcotráfico, el paramilitarismo, la guerrilla y la militarización de la región (Artunduaga, 1987; Marín, 2013). No se debe dejar a un lado la ampliación en la región de la economía extractiva del petróleo, practicada por el mismo Estado. Estas situaciones han conducido a que los koreguaje sean declarados un grupo nativo en vía de extinción física y cultural ${ }^{12}$. La lucha por la persistencia como grupo indígena ha adoptado durante la historia diferentes caminos, como lo han sido las migraciones, el adentramiento en la selva, la consolidación y reagrupamiento de identidades étnicas, el fortalecimiento y la revitalización lingüística y cultural, entre otros. Si bien no debe afirmarse que la consolidación de la identidad como grupo indígena y los procesos de resistencia para la persistencia como tal pasan necesariamente por la tenencia de una lengua propia, debe reconocerse que, para el caso de los koreguaje - cuya

12. Así lo reconoció la Corte Constitucional en el Auto 004 de 2009.

LINGÜÍSTICA Y LITERATURA 
lengua es hablada por la mayoría de ellos_-, esta ha sido un componente vital en el fortalecimiento de la identidad, la autodeterminación, la gobernanza y la soberanía como medios de resistencia al conflicto librado en su territorio. Ha sido la tradición oral la principal transmisora (Rodríguez, 2000). Sin embargo, se ha buscado fortalecer la lengua koreguaje con la adopción de la escritura, y en este proceso juegan claramente un papel los estudios realizados y la documentación lingüística sobre la misma.

Ahora bien, la historia del territorio ha tenido claramente consecuencias en reordenamientos, pérdidas y fusiones de elementos de la organización social, la manera de relacionarse con el territorio habitado, aspectos económicos, ideológicos y simbólicos, como también en reagrupamientos de «grupos etnolingüísticos». Estos acontecimientos han influido en las cualidades y la manera en que se ha realizado la documentación sociolinguiística en ciertos momentos dados.

La documentación sobre los koreguaje y su lengua es igualmente escasa. La mayoría de los documentos no se refieren a ellos de primera mano, sino en relación con otros grupos. De los primeros etnógrafos en mencionar el grupo de sociedades y lenguas de la región fue Von Martius, quien afirma que «en las zonas occidentales de las fuentes del Caquetá y del Vaupés, los indios Tamas han perdido entre otras hordas Coreguajes sedentarias, Amaguajés, Panenua que fueron antiguamente llamados terribles Antropófagos»(Von Martius, 1867, p. 560). Años antes de la publicación de Von Martius, Codazzi haría presencia en la región y elaboró una pequeña descripción de los koreguaje. De su lengua diría que es distinta a la de los guaguas, pudiéndose afirmar que es un dialecto de la lengua de los tamas. Manuel María Paz dibujó algunas acuarelas de los mismos que serían comentadas por L. M. J., de quien se dice fue un fraile misionero, estudioso y conocedor de la etnología colombiana y quien a su vez dejó un manuscrito del Diccionario Etnológico de Colombia del que sólo se conservó el apartado referente a la letra C (Díaz Piedrahita, 2013). En el manuscrito el fraile hace un breve recuento de los vestidos, los adornos, instrumentos, armas, fiestas y costumbres de estos. De igual manera, inserta un vocabulario de la lengua koreguaje publicado en 1855 recopilado por Manuel María Albis en Los indios del Andaquí. Memorias de un viajero, publicado por José Vergara y Evaristo Delgado.

Antes de estos documentos, los únicos que dan cuenta de la comunidad son aquellos relativos a las fundaciones que, en primera instancia, registraron tangencial e indiscriminadamente a las LINGÜÍSTICA Y LITERATURA 
etnias koreguaje, tama, carijona, andaquí, payaguaje y bayuguaje. Marín (2013) señaló que estas primeras referencias étnicas a estas agrupaciones - llámense naciones, tribus, parcialidades, pueblos - dan pistas sobre la composición de las mismas en relación con las reducciones de los agrupamientos, dadas en las encomiendas o reparticiones adjudicadas a los conquistadores. Constituyen estos registros las primeras aproximaciones al conjunto lingüístico tukanooccidental colombiano y al subgrupo que actualmente incluye a los koreguajes, sionas, tamas y macaguajes.

La documentación sobre los afluentes de los ríos Orteguaza y Caquetá quedó delegada a las misiones religiosas (jesuitas, franciscanos, y dominicos). No obstante, con la entrada de nuevos colonizadores, particularmente de caucheros quienes ejercieron una enorme presión sobre los poblados indígenas, esta documentación se reduce notablemente relegándose prácticamente a la realización de listados. Los misioneros van sustituyendo además la toponimia de los lugares por nombres de ciudades europeas: Florencia, Venecia, Milán y Puerto Asís son algunos ejemplos. Además, debe considerarse que la separación de Colombia, Ecuador y Perú, también influyó en la atomización de comunidades que siempre estuvieron intercomunicadas (Marín, 2013). Tales situaciones contribuyeron a generar un vacío documental y una incomprensión sobre los koreguajes, y los tukanos en general.

Las primeras referencias sobre estudios lingüísticos se encuentran en el trabajo del capuchino y lingüista Marcelino de Castellví, quien desarrolló en la región su labor investigativa y catequista, y fundó el Centro de Investigaciones Linguiísticas y Etnográficas de la Amazonia Colombiana CILEAC. El Handbook of the South American Indian (1948) de Steward reordenó la información etnográfica existente al momento, lo que permitió a Castellví (1955) catalogar la información disponible y publicarla con el CILEAC. En esta catalogación el koreguaje aparece referenciado entre las cuencas de los ríos Orteguaza y Yarí, con vestigios de chibcha. Pertenece, junto con el tama, a la subsección del Caquetá de la sección occidental de la familia tukano de Colombia. También, entre los primeros trabajos de estudio y catalogación de lenguas de la región se encuentra el Inventario de Lenguas y Dialectos Indígenas de Colombia (MacQouwn, 1955) y algunas recopilaciones de Rivet y Beuchat en Brinton (1892, citado por Rodríguez, 2000, p. 200). 
Posteriormente, es ampliamente conocido el trabajo desarrollado por investigadores del Instituto Lingüístico de Verano, que iniciaron el programa con los koreguaje en 1969 con el apoyo de Dorothy Cook y Carolyn Müller, siendo esta última reemplazada posteriormente por Frances Gralow. De esta etapa existe una buena cantidad de títulos que revelan, al menos, la relevancia que el Instituto Lingüístico de Verano tuvo dentro de la comunidad. En el texto Recuerdos y reflexiones: sobre la labor en Colombia (1962-2000) del ILV, se retoma una frase de Dorothy Cook en su misión con los koreguaje. Ella manifestó:

Cuando llegamos por primera vez al pueblo koreguaje de Maticurú explicamos a la gente que queríamos aprender su idioma, enseñarles a leer y a escribir en koreguaje y traducir las Sagradas Escrituras para ellos. El hijo del jefe se comprometió a enseñarnos y con frecuencia nos seguía por el pueblo diciéndonos palabras nuevas y nosotras las escribíamos en nuestros cuadernos. Después olvidamos algunas de las palabras y le preguntamos otra vez como expresar algo en su lengua y él nos decía: ayer les dije eso (Cook, 2000, p. 46).

Al inicio de la misión y por mucho tiempo, buena parte del material producido era de una naturaleza tal que se dedicó a la traducción y transcripción de palabras, como lo sugiere anteriormente Dorothy Cook, razón por la cual existe buena cantidad de vocabularios de la lengua. Con el tiempo los investigadores del ILV profundizaron más en la gramática de la lengua y, finalmente, fruto de esa misión, se publicaron, según el ILV:

Un abecedario, una cartilla para preescolar, seis cartillas para la alfabetización, una cartilla para transición, veinte títulos en la lengua vernácula sobre la salud, la ciencia, la cultura koreguaje u otra cultura y la historia colombiana, y veintiún libros escritos por autores koreguajes sobre sus experiencias o sus leyendas. En estudios etnolingüísticos se publicó un artículo sobre aspectos de la cultura material, un artículo sobre el parentesco, dos títulos sobre la fonología, cinco títulos sobre la gramática, seis títulos sobre la lingüística textual, un diccionario escolar, un diccionario de frases y un diccionario de todos los datos. En obras de traducción se publicó un resumen del Antiguo Testamento, el Nuevo Testamento en koreguaje y un himnario (Frank, 2000, pp. 46-47).

Dicho material permite acercarse, en primera instancia, a la lengua koreguaje como hablantes del español y desde una perspectiva lingüística que la ha ceñido a las categorías creadas por esta y que, usadas en el aula de clase y en otros ámbitos ha permitido que en los últimos años se realice un mayor énfasis en la escritura de la lengua. Por ello es paso obligatorio la revisión de lo escrito por el ILV a la hora de entender la grafía en koreguaje. En esa escritura reciente resaltan notablemente los temas a tratar en el texto Experiencias de personajes Koreguajes del año 2000, compilado por Frances Gralow y Juan Pizarro Gutierrez, en la cual se describen los aspectos de 
la realidad de la comunidad y de sus vivencias personales, como las anécdotas sobre el río, en la chagra, en las horas de caza, así también acerca del conflicto armado, de los viajes a Lomalinda, de la estación del ILV, de la escritura de libros, etc. Igualmente, el libro trasciende más allá del presente de los koreguajes al grabar en papel parte de su memoria colectiva, inscrita en su tradición oral y en sus relatos míticos.

Algunas investigaciones de corte académico y científico han sido realizadas por el profesor Pedro Marín en calidad de asesor del CRIOMC en representación de la Universidad Nacional de Colombia, las cuales han permitido recoger una serie de relatos que dan cuenta del recorrido de los héroes culturales de los koreguaje a través del territorio ancestral, dada la importancia que el Ministerio de Educación le concedió a la investigación de cosmologías y sistemas de referencia ideológica propias de la comunidad en proyectos etnoeducativos. Lo anterior se dio debido a que estos temas ayudan a constituir el primer capítulo de la historia de estos pueblos concerniente a la historia de sus ancestros y su mitología.

Durante la década de los ochenta se realizaron algunas tesis de pregrado en temas lingüísticos de la lengua koreguaje. Esta serie de trabajos fueron realizados en el marco de las prácticas de Fonética y Fonología Comparada y Seminario Etnolingüístico de la anterior carrera denominada Filología e Idiomas de la Universidad Nacional de Colombia. Las prácticas fueron llevadas a cabo en las comunidades koreguaje de San Francisco, Agua Negra, San Luis y Maticuru, bajo la dirección investigativa de los profesores Carlos Dupont y Pedro Marín. Algunos de los objetivos investigativos comprendían el estudio de la morfología nominal en lengua koreguaje, la asignación de un sistema de escritura, la manifestación de la visión cosmológica en la lengua, la educación bilingüe y la aproximación morfológica al sistema verbal en la misma lengua. Entre algunos de estos trabajos se encuentran Aspectos fonológicos de la lengua coreguaje de Cristancho y García (1982), un estudio que brinda algunas aproximaciones al sistema fonológico: las consonantes, las vocales y el acento suprapragmático. Señaló este último autor, que, dada la situación de la lengua en contacto con el español, la glotalización tiende a desaparecer, presentándose la duplicación vocálica.

No se han llevado a cabo trabajos etnográficos en profundidad entre los koreguaje. No obstante, en el Departamento de Antropología de la Universidad Nacional de Colombia, reposan dos tesis de pregrado de la década de los noventa que abordan el proceso de socialización de los 
koreguaje (Quiroga Zuluaga, 1997) y la organización social centrándose en el proceso de reconstrucción como comunidad (Álvarez Contreras, 1995). Igualmente, se encuentran algunas investigaciones lingüísticas, realizadas tras el interés que produjeron en los estudiantes las inmersiones propuestas por Dupont o por Marín, en las cuales se reseña brevemente información etnográfica relativa a la vida en la comunidad a modo de introducción y contextualización histórica.

En la actualidad, y según el informe mencionado anteriormente del Ministerio de Cultura, la comunidad hace uso de algunos materiales escritos que tiene en su poder. Entre los títulos se encuentran: Textos en koreguaje, de Pedro Marín Silva; Ko'rehuaj ch'ore cutuñu (Hablemos coreguaje: Un libro de aprendizaje del idioma coreguaje, tomo 1), de Frances Gralow y Dorothy Cook, del Instituto Lingüístico de Verano; Cuando no había agua y otras leyendas indígenas, de Janet Barnes, del ILV; Diccionario bilingüe koreguaje-español / español-koreguaje, de Frances Gralow y Dorothy Cook del ILV; Formas de tematización en coreguaje, de Frances Gralow del ILV; Lista de palabras swadesh y rowe y Un bosquejo del idioma koreguaje, de Frances Gralow, del ILV. Igualmente, son cada vez más las iniciativas nacidas desde la propia comunidad para incentivar, no sólo la apropiación de la lengua como elemento identitario, sino también de otro tipo de prácticas relevantes para la comunidad como lo son las actividades productivas en los espacios destinados para tal fin (la vega, la chagra, el cananguchal, la montaña, el río, la laguna, el caño, etc.) que permiten, además, la realización de cartografías sociales, calendarios productivos, etc. lo que hace constar de la íntima relación que mantienen con su territorio.

En 2007, como resultado del Programa de Concertación y Estímulos del Ministerio de Cultura, el resguardo indígena La Esperanza del municipio de Milán (Caquetá) realizó una publicación en la cual se transcriben únicamente en lengua koreguaje algunos cantos e historias de origen. Solamente los nombres de los cantos cuentan con traducción al español. Entre algunos de estos se encuentran: «Árbol del agua» (okosukinu), «Canto de la muchacha diabla» (jurebatiromio), «Canto de las mujeres negras» (chiromi), «Canto del pez mojarra» (konobaniruru), «Canto del baile de perfume de plantas» (bi-mañafire), «Canto del baile del ave» tente (tuntu-uja), «Canto del baile del cacao» (nabamairi), «Canto de baile de la culebra» (añabeñe), «Canto de un personaje malo para la caza y pesca» (baijñama-ai), «Canto de los 
indígenas borrachines» (konobaju pai), «Canto del baile del coco» (peto soaja), y «El origen del alimento» (au inasie).

Respecto a la documentación audiovisual, la Biblioteca Nacional tiene en su poseer un par de grabaciones realizadas por la Misión Cultural Técnica Holandesa, específicamente por Bernardo Broere y Sylvia Moore, que datan de 1978, y que hacen parte del Primer Festival Musical Indígena del Sur en el que un grupo koreguaje de Aguas Negras (Caquetá) realizó una participación. De este festival existen tres audios titulados «Cantos koreguajes», los cuales no cuentan con descripción alguna y de los que se desconoce el tema a tratar dada la distancia que existe respecto a la lengua. En estas grabaciones las voces son los instrumentos del grupo, mezcladas con el sonido de tambores, capadores y carrizos. En otro de los audios de dicha jornada, titulado «Música koreguaje», se nota la melodía de flautas de pan y tambores. Existe, además, un audio adicional, de nombre «Tente», el cual está dividido en cinco partes y es acompañado con un rondador, una caja y un firisai. Su letra habla de un ave que habita exclusivamente en la Amazonia: el tente, cuyo sonido es grave fuerte y resonante. Dicha ave es domesticada por los grupos indígenas para que les sirva de guardián. Es muy apreciada, además, porque se le atribuyen capacidades para espantar y cazar serpientes.

Existe igualmente una serie de grabaciones del 18 de noviembre de 1989, elaboradas por José Leonte Plaza Murcia, en el marco de la Jornada Regional de Florencia (Caquetá). Dichas grabaciones tienen los títulos «Vengo a hacer una música indígena», «Estoy tomando» y «Pájaro picón-Yataro». Todas ellas fueron interpretadas con capadores, carrizos y tambores.

En producciones más recientes existe una serie llamada «Cosmogonías», realizada por el Canal Trece, la cual consta de cuatro capítulos con una duración de aproximadamente 27 minutos cada uno, que a su vez recogen temas relevantes de la cosmovisión de los koreguaje, como algún breve relato de un mito de origen, las vivencias de la violencia por el conflicto armado, la organización a través de un plan de vida, el papel de la escuela dentro de la comunidad, etc. Por otra parte, se cuenta con documentación de audio realizada en el marco de un curso de Lingüística Antropológica en la Universidad Externado de Colombia, Facultad de Ciencias Sociales y Facultad de Ciencias de la Comunicación por la catedrática Consuelo De Vengoechea y el entonces estudiante koreguaje Alirio Lozano en el año 2014. 
Es también destacable la publicación de 2014 titualda De agua, viento y verdor: Paisajes sonoros, cantos y relatos indígenas para niños y niñas, realizada por el Instituto Colombiano de Bienestar Familiar en asocio con el Ministerio de Cultura y varias fundaciones, en la cual se incluye un archivo sonoro de 16 pistas Korebaju, dirigidos a la infancia como recurso para el fomento y fortalecimiento de la lengua.

Los anteriores materiales son relevantes a nivel de la comunidad, en tanto valoriza los conocimientos y los contenidos propios. Gracias a los recursos tecnológicos modernos se puede almacenar la información mencionada anteriormente en un formato que permita su conservación y reproducibilidad, la cual puede resultar llamativa para las nuevas generaciones. Hay que tener en cuenta que tales formatos poseen la capacidad de motivar a las personas a interesarse por temas que no le son ajenos y sobre los cuales resulta pertinente realizar un acercamiento.

\section{Contradicciones y retos de la revitalización lingüística y cultural}

Este artículo, como se mencionó en un principio, parte de la necesidad de dar relevancia a los pocos estudios que existen sobre dos pueblos indígenas amazónicos, dos lenguas poco documentadas y la necesidad de crear de manera colaborativa nueva documentación etnográfica y lingüística que pueda ser útil tanto en el conocimiento existente sobre estos grupos, como en las reivindicaciones sociales y de fortalecimiento cultural, social y lingüístico. Dentro de ese proceso, y pensando en miras a un proyecto de revitalización lingüística y cultural, se desea indagar en lo que respecta a las contradicciones con las que muchas veces se encuentra.

La primera pregunta que se formula siempre a la hora de llevar a cabo un proyecto de revitalización lingüística es ¿cómo empezar? David Crystal en su libro Language death en un apartado del mismo nombre afirma lo siguiente:

[...] existen suficientes casos de estudio en el mundo de lenguas que han experimentado un renacimiento y que muestran que la pérdida no es siempre inevitable. Es mucho lo que pueden hacer y ya han hecho las comunidades indígenas, los grupos de apoyo local y los organismos externos (Crystal, 2001, p. 107).

Sin embargo, las experiencias en nuestro país son reducidas, y en la Amazonia aún más. En esta última existen unas características particulares que han de ser tenidas en cuenta a la hora de 
ejecutar cualquier proyecto. Por tal motivo, se hace referencia brevemente a un par de procesos que se han llevado a cabo en Colombia, específicamente con los nonuya en el Amazonas, con los nasa en el Cauca y con los kankuamos en la Sierra Nevada de Santa Marta, con el fin de establecer esas contradicciones que surgieron en cada uno de los procesos y que responden a condiciones concretas de cada uno de ellos.

Lo primero es establecer y reconocer la documentación con la que se cuenta y partir de allí crear el corpus revitalizador. Al ahondar en esa historia de documentación de la lengua nononotá, lo primero que se percibió es que, aunque los antecedentes del proyecto se remontan varias décadas atrás, los esfuerzos interrumpidos impidieron que el proceso se consolidara, de modo que no se frenó la tendencia a que la lengua nativa deje de enseñarse a niños y jóvenes en la mayoría de los hogares, dejando de lado así la transmisión intergeneracional de estas. Las razones, en el contexto amazónico, de esta situación son varias y de muy distinta índole. En el caso de los muinane, alrededor de los años noventa, los niños asistían al internado de Araracuara, razón por la cual dejaban su comunidad buena parte del año, impidiendo así el proceso intergeneracional de transmisión de conocimientos en la edad temprana. A su regreso a la comunidad hablaban poco su lengua y permanecían, además, entonando cánticos religiosos que allí les fueron enseñados. Hoy en día, aunque existe ya una escuela en la comunidad, en ella se ofrece sólo hasta grado quinto de primaria, por lo que al llegar a este grado el proceso se trunca, al igual que antes, ya que para continuar los estudios se han de desplazar a Puerto Santander o a Araracuara. Por lo general, entre estas razones se encuentra que, como lo describió Mahecha (2011, p. 304) el aprendizaje de una lengua como el español permite a los jóvenes indígenas acceder a los conocimientos del mundo blanco y les brinda la posibilidad de practicar relaciones políticas más equitativas. Además, representa un mecanismo de ascenso social que amplía las opciones laborales y el acceso a posiciones de poder y autoridad, aunque muchas veces en detrimento de la cultura propia. En este sentido, es importante mencionar la influencia que tuvo la bonanza cauchera en el debilitamiento cultural de la llamada Gente de Centro. Uno de los principales problemas que estas lenguas enfrentan es el número de hablantes y el contexto en el que estas se usan. En innumerables ocasiones, al ser ya tan reducido el uso de las lenguas en cuestión, se supone prácticamente la extinción de estas a mediano plazo por más esfuerzos que se hagan.

LINGÜÍSTICA Y LITERATURA 
De esta manera, en cualquiera de los casos, la situación que se afronta cuando se decide trabajar para frenar - $\mathrm{O}$ al menos desacelerar-, el proceso de pérdida de la diversidad lingüística resulta paradójica, pues las lenguas en estado de vulnerabilidad como lo son las amazónicas — para hablar del caso particular de este estudio_-, como lo señaló Crystal (2001), morirán a menos que se haga algo al respecto. Pero la realidad es que también, pese a que se actúe, pueden morir de cualquier manera. Esta situación obliga a tomar una actitud mucho más amplia frente al trabajo en pro de estas lenguas, que desborde el ámbito meramente lingüístico por el riesgo inminente de su desaparición, y este mucho más involucrado al fortalecimiento de la soberanía política y los derechos sociales y ambientales perseguidos como colectividad indígena. Esta situación se repite a lo largo y ancho del globo y en el caso de nuestro país se acentúa en distintos grupos, por citar un caso particular, el caso kankuamo, en la Sierra Nevada de Santa Marta, cuya lengua no se habla en la región pero que, según las tradiciones locales, ciertos mamos kogi poseen aún este conocimiento en las regiones apartadas de la sierra, debido al proceso histórico de asimilación y mestizaje al que se vio abocado el pueblo kankuamo desde muy temprano en su encuentro con la sociedad occidental, que los llevó a hacer entrega de su conocimiento a los mamos kogi con el fin de resguardarlo. Lo que resulta notable para este caso es que hoy en día se habla de un retorno de los kankuamo, por medio de la persistencia en el tiempo de tradiciones y conocimientos que permiten materializar un proceso de resistencia y de unificación como comunidad y que supone una instrumentalización de la identificación étnica, ya no como un «descubrimiento de experiencias históricas y tradiciones culturales, sino como un proceso en el cual estas dimensiones son movilizadas como recursos culturales en coyunturas particulares» (Morales, 2011, p. 155). Estos «idiomas de la reetnización»son, en cierto sentido, una reconstrucción de la relación con los ancestros en base a la memoria que de ellos ha quedado en fiestas, sitios de pagamento, versos, etc., las que permiten a su vez un redescubrimiento de esa memoria que se ha reproducido corporal y espacialmente, sobre la cual se sustenta parte del proyecto actual de fortalecimiento cultural. Aunque la lengua ha quedado relegada dentro de este proceso, se continúa reflexionando en torno a la importancia de esta, aun cuando el material existente continúa siendo escaso, bastante especializado para lingüistas y poco divulgado a las nuevas generaciones. 
Queda claro que, aunque por lo general los proyectos de revitalización de las lenguas inician con un estudio sobre la situación de las lenguas y la documentación de las mismas, este resulta ser apenas un paso básico a la hora de determinar el estado de vitalidad de las lenguas, así como las acciones necesarias a emprender para su revitalización. Tales acciones deberán desarrollarse en un contexto propicio, en el cual sean los propios hablantes y la comunidad quienes asuman pública y colectivamente una actitud positiva frente al fortalecimiento de su propia lengua, lo cual resulta ser una dimensión necesaria que desborda por completo los alcances de una investigación lingüística y académica sobre una o varias lenguas. Por obvio que parezca, la obligatoriedad de esta situación para el desarrollo de una investigación y de un proyecto de fortalecimiento lingüístico, la observación que se hace implica entender las razones por las cuales la mayoría de las lenguas indígenas se encuentren en una serie de circunstancias históricas que las han llevado a encontrarse en un espacio de disputa y de conflicto donde los intereses en ellas y en sus procesos de fortalecimiento pasan por varios altibajos.

Para el caso de la Amazonia y, concretamente de los muinane, se evidencia una necesidad de agrupación o unificación de los esfuerzos de las comunidades que se encuentran altamente dispersas en el territorio en torno a este tipo de proyectos, que han de ser ejecutados con un alcance no sólo local, sino también regional, y que tenga además como uno de los ejes claves el entorno familiar. Ximena Pachón (1997) señaló que, para el caso del pueblo nasa, el grado de conservación de su lengua es mayor en la medida en que la estructuración del núcleo familiar se realiza en torno a la figura de los abuelos, quienes influyen a su vez para que la constitución doméstica se estructure por familias extensas y no familias nucleares extendidas. De igual manera, resulta importante la competencia lingüística de la madre en la lengua nasa para que en el proceso de socialización esta sea aprendida por los niños. Dicha apreciación es posible de ser traspolada al caso muinane, que en las últimas generaciones ha cambiado la manera en que se relacionan entre ellos los padres e hijos.

Las dificultades de los proyectos mencionados anteriormente para llegar a los ámbitos privados suponen que, como afirmó David Crystal, el meollo principal del asunto sea «que las personas desarrollen el sentido del valor que posee una lengua, y de lo que se pierde cuando muere una lengua» (2011, p. 114) para que los esfuerzos puedan trascender los intereses de los investigadores y se conviertan también en los intereses de la comunidad y de las familias. Así, si 
se logra desarrollar ese sentido del valor de la lengua, puede ser que el proyecto llegue a buen puerto en cuanto los investigadores se retiren de la comunidad. No obstante, hay que anotar la fuerte tendencia de este tipo proyectos por estimular los formatos escritos en lenguas indígenas que han perdurado a lo largo del tiempo gracias a la transmisión oral de las mismas. Dicha contradicción supone que en los proyectos de revitalización se privilegien espacios distintos a los de la escuela, en donde siempre se han enfocado este tipo de proyectos. Un contexto privilegiado en el que las lenguas son usadas en las sociedades amazónicas son los bailes, dentro de los cuales están los que se hacen en honor a las frutas, en las celebraciones de los bautizos, en los rituales de cacería, o en los de tablón. En dichos bailes, se deben cantar las canciones tanto en la lengua de los anfitriones como en la de los invitados. Así, los cantos son un recurso didáctico por excelencia para la enseñanza de la lengua a los más jóvenes que, en el caso de los nonuya, fueron usados a la hora de seleccionar y crear corpus revitalizadores (canciones, narraciones y léxico) tales como los cantos inspirados en los compuestos por los vecinos de otros grupos, solo que a partir de la fauna y flora local.

Esta metodología se realizó en conjunto con la comunidad nonuya bajo el supuesto de que el trabajo común permite el reconocimiento de la lengua minoritaria y, asimismo, suscita tanto el interés en ella como su reapropiación. A través de la recopilación, transcripción, ilustración, diseño de materiales, entre otras actividades se revalora la lengua individual y socialmente. Entre otras cosas, Isabel Romero — quien se apoyó en otros procesos de revitalización—, afirmó que la intervención con fines documentales y el proceso de reversión del desplazamiento tienen un efecto positivo en la comunidad:

Genera curiosidad que quien investiga, que hace parte de la sociedad mayoritaria, se interese por aprender la lengua que sólo saben los mayores. Esa presencia es inquietante, pero puede ser el motor para la reversión de los procesos de desplazamiento lingüístico y cultural. Así se fortalecen el autoconcepto y la percepción de la relevancia de lo propio (Romero, 2015, p. 58).

Las actitudes de los hablantes hacia las lenguas son factores desencadenantes del cambio. Así, el abandono de la lengua es casi una adaptación a un ambiente en donde el uso de esta no es más beneficioso para ellos. Pachón (1997), afirmó que la escuela fue uno de los factores que atentaron contra la lengua nasa, dado que a través de esta se instauró la idea del español como lengua civilizada, llegando incluso a fomentarse el desprecio y la mofa de la lengua materna. 
No muy distinto resultó el papel que jugaron los internados dentro de las sociedades amazónicas. Para revertir dicha situación, los procesos de revitalización lingüística se han preocupado por institucionalizar su enseñanza en el ámbito escolar, relegando en ocasiones los ámbitos cotidianos. Sin embargo, no se puede renunciar tampoco a la escuela como lugar de la revitalización lingüística, en tanto esta, como ámbito educativo, hace también parte de la lucha política y de la autonomía de las comunidades indígenas.

Múltiples pueblos indígenas han vivido de distintas maneras la inmersión de la violencia en sus territorios, lo que ha llevado en detrimento entre tantos aspectos, a sus lenguas. Resulta ser axiomático que el bienestar físico es y debe ser prioritario frente a cualquier otro fin, especialmente la conservación y el fortalecimiento de su propia lengua. No obstante, lo dicho debe interpretarse desde las particularidades de cada comunidad. En algunas de ellas, tanto la lengua propia como el reagrupamiento y fortalecimiento en torno a una identidad étnica se han configurado como herramientas para la persistencia física de la comunidad en condiciones adversas, consolidándose como un fin común a perseguir. Pero esto no es una situación homogénea en todos los pueblos indígenas, pues muchos de sus integrantes consideran que la entrada a la economía de mercado, al menos en apariencia, logrará de manera mucho más efectiva su pervivencia física, lo cual es algo que la lengua mayoritaria y los valores que se le ha atribuido les permite de igual manera el progreso, la equidad y el acceso a oportunidades educativas y laborales.

Por lo general, en estas condiciones, aunque se reconozca la riqueza cultural de la lengua y su inmenso universo de significado, por lo menos en retórica, el involucramiento personal para la transmisión a futuras generaciones es débil. De allí la importancia de que el trabajo por el fortalecimiento de una lengua se desarrolle de diversas maneras y perspectivas, de tal forma que se involucre no solo con el componente lingüístico y político de hablar la lengua propia, sino también apele a una dimensión personal, emotiva, ecológica, y que puedan las personas hallarle sentido y lugar en sus proyectos de vida.

\section{Referencias bibliográficas}


1. Albis, M. M. (1855). Los indios del Andaquí: memorias de un viajero publicadas por José $\mathbf{M}^{\mathrm{a}}$ Vergara i Vergara i Evaristo Delgado. [S.1.] [s.n.] Popayán: Imprenta de La Matricaria.

2. Arango, R. y Sánchez, E. (2004). Los pueblos indígenas de Colombia en el Umbral del Nuevo Milenio. Departamento Nacional de Planeación. Bogotá D.C.

3. Artunduaga, F. (1987). Historia general del Caquetá. Florencia: Fondo Mixto para la Promoción de la Cultura y las artes del Caquetá.

4. Aschmann, R. (1993). Proto Witotoan. Dallas: The Summer Institute of Linguistics at the University of Texas at Arlington.

5. Barnes, J. (1993). Cuando no había agua y otras leyendas indígenas. Asociación Instituto Linguistico de Verano. Santa Fe de Bogotá: Editorial Alberto Lleras Camargo.

6. Cabildo Indígena de la Esperanza (Caquetá). (2007). Rescate de la tradición oral: pueblo indígena Koreguaje. Milán (Caquetá): Ministerio de Cultura.

7. Castellví, M. (1955). Propedéutica Etnioglotológica y Diccionarios Clasificador de las lenguas Indoamericanas. Madrid: Consejo Superior de Investigaciones lingüísticas. Instituto Bernardino de Sahagún.

8. Chukíki. Consejo de Ancianos de los tres grupos muinanes. (1994). Un Alfabeto para el Pueblo Muinane. Marco cultural del proyecto de investigación para la elaboración del alfabeto. Predio Putumayo, Amazonas: Manuscrito.

9. Chukíki. Consejo de Ancianos de los tres grupos muinanes. (2010). Censo general de la Gobernación Muinane Chukíki. Predio Putumayo, Amazonas. (Inédito).

10. Cook, D. (2000) Koreguaje. Recuerdos y reflexiones. En P. Frank, (Ed.), Recuerdos y reflexiones: la AILV y su labor en Colombia (1962-2000). (pp. 46-47). Asociación Instituto Linguistico de Verano. Bogotá: Editorial Alberto Lleras Camargo.

11. Crystal, D. (2000). Language Death. Cambridge: University Press.

12. Díaz Piedrahita, S. (2013). Un curioso manuscrito etnográfico. Revista Académica Colombiana de Ciencia, 37(143), 159-176.

13. Dixon, R. M.; Aikhenvald A. (Eds.) (1999). The Amazonian Languages. Cambridge: University Press.

14. Dupont, C. (1982). Clasificación nominal en la lengua Koreguaje. Forma y Función, [S.1.], 4, $35-46$.

LINGÜÍSTICA Y LITERATURA

ISSN 0120-5587

E-ISSN 2422-3174

N. ${ }^{\circ} 76,2019,17-45$ 
15. Echeverri, J. A. (1992). Sondeo de fuentes bibliográficas sobre lenguajes del interfluvio Caquetá-Putumayo, Amazonía colombo-peruana. Amerindia, 17, 149-172.

16. Echeverri, J. A. (1997) The People of the Center of the World: A Study in Culture, History, and Orality in the Colombian Amazon. (Ph.D. Dissertation), New School for Social Research, New York.

17. Echeverri, J. A. (2016). Fééne fívo játyime iyáachimihai jínije. Territorio primordial de vida de la descendencia del Centro: Memorias del territorio del Pueblo Fééneminaa Gente de Centro. Bogotá: Impresol Ediciones.

18. Echeverri, J. A.; Landaburu, J. (1995). Los nonuya del Putumayo y su lengua: Huellas de su historia y circunstancias de un resurgir. En M. Pabón Triana (Ed.), La recuperación de lenguas nativas como búsqueda de identidad étnica. (pp. 39-74). Bogotá: Universidad de los Andes, CCELA.

19. Frank, P. (2000). Recuerdos y reflexiones: la AILV y su labor en Colombia (1962-2000). Asociación Instituto Lingüístico de Verano. Santa Fe de Bogotá: Editorial Alberto Lleras Camargo.

20. Gómez López, A. J. (2010). El Regreso de las misiones: memoria de la primera excursión apostólica capuchina al territorio de los «infieles huitotos». Manuscrito.

21. Gralow, F y Cook, D. (1984). Ko'rehuaj ch'ore cutuñu (Hablemos coreguaje: Un libro de aprendizaje del idioma coreguaje, tomo 1). Instituto Lingüístico de Verano. Santa Fe de Bogotá: Editorial Townsend.

21. Gralow, F. (1969). Lista de palabras swadesh y rowe y Un bosquejo del idioma koreguaje. Santa Fe de Bogotá: Instituto Lingüístico de Verano.

22. Gralow, F. (1980). Formas de tematización en coreguaje. Instituto lingüístico de Verano. Santa Fe de Bogotá.

23. Gralow, F. y Cook, D. (2001). Diccionario bilingüe koreguaje-español / español-koreguaje. Bogotá. Editorial Alberto Lleras Camargo.

24. Instituto Lingüístico de Verano y Ministerio de Gobierno-República de Colombia (Eds.) (1981). Estudios en andoke y muinane. (Traducción española de Casas, R. y Pombo, J. M.) Lomalinda: Townsend. 
25. Koch Grünberg, T. (1909). Zwei Jahre unter den Indianern Reisen in Nordwest-Brasilien 1903-1905. Berlin: Wasmuth. Traducción española: Dos años entre los indios: viajes por el Noroeste brasileño 1903-1905 (1995) por Watzke, A., Camacho, R., Ortiz, M. M. y Castillo, F. Vol. 2. Bogotá: Editorial Universidad Nacional de Colombia.

26. Landaburu, J. (Ed.) (1996). Documentos sobre lenguas aborígenes de Colombia del archivo de Paul Rivet. Lenguas de la Amazonía colombiana. Vol. 4. Bogotá: Universidad de Los Andes. 27. Landaburu, J. (2000). Clasificación de las lenguas indígenas de Colombia. En M. S. González de Pérez y M. L. Rodríguez de Montes (Eds.), Lenguas indígenas de Colombia: una visión descriptiva (pp. 25-48). Bogotá: Instituto Caro y Cuervo.

28. Llanos, H. y Pineda Camacho, R. (1982). Ethohistoria del gran Caquetá. Siglos XIV-XIX. Bogotá: Banco de la República.

29. Londoño Sulkin, C. D. (2004). Muinane. Un proyecto moral a perpetuidad. Medellín: Universidad de Antioquia.

30. Loukotka, C. (1968). Classification of South American Indian Languages. En J. Wilbert, (Ed.). California: University of Los Angeles.

31. MacQouwn, N. (1955). The Indigenous Languages of Latin America. American Anthropologist, 57, Lancaster University Press.

32. Mahecha, D. (2011). Escuela y multilingüismo en Amazonia: un desafío contemporáneo. En Cátedra Jorge Eliécer Gaitán. Amazonia colombiana. Imaginarios y realidades. Bogotá: Universidad Nacional de Colombia, 293-310.

33. Marín Silva, P. (2013). Introducción histórica a la etnia Coreguaje. Revista Brasileira de Lingüística Antropológica, 5(1), 193-215.

34. Martius, K. F. P. (1867). Beiträge zur Ethnographie und Sprachenkunde Amerikas zumal Brasiliens, I. Zur Ethnographie. Leipzig: Friedrich Fleischer.

35. Minor, E. E. y de Minor, H. (Comps). (1971). Vocabulario muinane y huitoto. Yarinacocha: Instituto Lingüístico de Verano.

36. Morales, P. (2011). Los idiomas de la reetnización. Corpus Christi y pagamentos entre los indígenas kankuamos en la Sierra Nevada de Santa Marta. Bogotá: Universidad Nacional de Colombia. 
37. Nejedeka, A. (2011). La ciencia de vida escrita en las aves Primera parte «Mookaani fiivo» Creación de nuestro Abuelo tabaco-vida de centro. Lengua Muinane. (Traducción de Célimo Nejedeka). En Mundo amazónico (pp. 329-357).

38. Pachón, X. (1997). El nasa yuwe o la lucha por la supervivencia de una lengua dominada. En Lenguas amerindias. Condiciones sociolingüísticas en Colombia. Santa Fe de Bogotá: Instituto Caro y Cuervo.

39. Pineda Camacho, R. (2000). Holocausto en el Amazonas. Bogotá: Editorial Planeta.

40. Rodríguez, S. (2000). Estudios sobre la lengua koreguaje. En Lenguas Indígenas de Colombia: una visión descriptiva (pp. 199- 213). Bogotá: Instituto Caro y Cuervo.

41. Romero Cruz, I. V. (2015). Lengua nonuya y conservación lingüística: algunas reflexiones. Mundo Amazónico, 6(2), 49-65.

42. Seifart, F. y Echeverri, J. A. (2015). Proto Bora-Muinane. Liames, 15(2), 279-311.

43. Silva, P. M. (2004). Mítica Korebajü. Colección Textos.

44. Silva, P. M. (2014). Archivo y semántica: etnohistoria de los guajes del piedemonte subandino amazónico colombiano, 27, 115-134. https://doi.org/10.15446/fyf.v27n2.47668

45. Silva, P. M. (n.d.). Fragmentos de mitología Coreguaje. Maguaré, 137-161.

46. Steward, J. (1948). The Handbook of the South American Indians. Washington D.C.: Smithsonian Institution. Bureau of American Ethnology. Bulletin 143.

47. Tobón, M. A. (2008). «La mejor arma es la palabra». La Gente de centro-kigipe urúki y el vivir y narrar el conflicto político armado. Medio río Caquetá-Araracuara, 1998-2004. (Tesis de maestría). Universidad Nacional de Colombia.

48. Tovar, A. y Larrucea, C. (1984). Catálogo de las lenguas de América del Sur. Madrid: Gredos.

49. Useche Losada, M. (1994). La colonia Penal de Araracuara. Socioeconomía y recursos naturales. Bogotá: Instituto Colombiano de Antropología.

50. Useche Losada, M. (1998). La prisión del Raudal: Historia oral de la Colonia Penal de Araracuara Amazonia Colombiana 1938-1971. Bogotá: Instituto Colombiano de Antropología.

51. Vengoechea, C. (1995). La dimensión aspecto temporal en muinane. (Tesis de maestría). Universidad de Los Andes. Bogotá: CCELA.

LINGÜÍSTICA Y LITERATURA

ISSN 0120-5587

E-ISSN 2422-3174

N. ${ }^{\circ} 76,2019,17-45$ 
52. Vengoechea, C. (1996a). Comentarios sobre los datos de la lengua muinane recogidos por Tastevin en 1920 y datos actuales. En J. Landaburu, (Ed.). Documentos sobre lenguas aborígenes de Colombia del archivo de Paul Rivet (pp. 553-561). Lenguas de la Amazonía colombiana. Bogotá: Universidad de Los Andes.

53. Vengoechea, C. (1999). Fonología de la lengua muinane. Memorias Congreso de Lingüística Amerindia y Criolla, 6, Bogotá: CCELA, Universidad de Los Andes, (pp. 17-46).

54. Vengoechea, C. (2001). Muinane y su sistema de clasificación nominal: interacción entre sintaxis y pragmática. Seminario CLANLINC: Clasificación Nominal en las Lenguas Amazónicas. Laboratoire Dynamique du Langage. Lyon: Université de Lyon 2. Inédito.

55. Vengoechea, C. (2004-2005). Morphosyntax of Muinane. Amerindia, 29/30, 119-140.

56. Vengoechea, C. (2003). Análisis del nombre a partir del léxico del cuerpo humano en muinane. Forma y Función, 16, 285-291.

57. Vengoechea (2012). Catégorisation lexicale en muinane Amazonie colombienne. Tesis doctoral. Publicación en la Red en los Archivos Abiertos de Tesis doctorales de Francia. Universidad de Toulouse II: Le Mirail.

58. Vengoechea, C. (2014). Categorías léxicas y clasificación nominal en muinane. Noroeste Amazónico. Revista Brasilera de Lingüística Antropológica de la Universidad de Brasilia, 6(1), 63-86.

59. Vivas, S. (2012). Kirigaiai: Los géneros poéticos de la cultura minika. Antípoda. Revista Antropología y Arqueología, 15, 223-244.

60. Walton, J. (1977). Participant Reference and Introducers in Muinane Clause and Paragraph. En R. Longacre, (Ed.). Part 3, 45-65. Arlington: Summer Institute of Linguistics-University of Texas. Traducción española: Sistemas de referencia al participante e introductores en la cláusula y el párrafo muinane. (1981) por Pombo, J. M. En: Estudios en andoke y muinane. Instituto Lingüístico de Verano y Ministerio de Gobierno-República de Colombia, (pp. 129-179). 61. Walton, J. \& Walton, J. (1967). Phonemes of Muinane. En V. G. Waterhouse, (Ed.), Phonemic Systems of Colombian Languages. Summer Institute of Linguistics of the University of Oklahoma. (pp. 37-47). Oklahoma: Norman. 
62. Walton, J., Hensarling, G.; Maxwell M. B. (2000). Muinane. En M. S. González de Pérez y M. L. Rodríguez de Montes, (Eds.), Lenguas indígenas de Colombia: una visión descriptiva. Bogotá: Instituto Caro y Cuervo, (pp. 255-273).

63. Walton, J., Pakky, C. y Staly, A. (1978). Estudios en andoke y muinane. Ministerio de Gobierno, Instituto Lingüístico de Verano. Lomalinda: Townsend.

64. Walton, J. \& Walton, J. (1967). Phonemes of Muinane. En Waterhouse, V.G. (Ed.). Phonemic Systems of Colombian Languages. (pp. 37-47). Summer Institute of Linguistics of the University of Oklahoma. Oklahoma: Norman.

65. Walton J. W., Walton J. y Pakky, C. (1997). Diccionario bilingüe muinane-español, español muinane. Bogotá: Editorial Alberto Lleras Camargo. 